\title{
Comparison of inbred lines extracted from two single crosses and their double cross by single seed descent
}

\author{
H. S. Pooni, \\ J. L. Jinks and D. Yohannes
}

Department of Genetics, University of Birmingham, Birmingham B15 2TT, U.K.

Three random samples of inbred lines, two $(B$ and $D)$ extracted from the $V 1 \times V 5$ and $V 2 \times V 12$ crosses of $N i c o t i a n a$ rustica and the third $(D c)$ produced from their double cross $(V 1 \times V 5) \times(V 2 \times V 12)$, have been compared for seven quantitative characters. Theoretically, the genetical expectations of the first, second, third and fourth order statistics of these samples are expected to differ both in the absence and presence of complications like epistasis, linkage disequilibrium and genotype $x$ environmental interactions. However, the magnitudes of these differences depend largely on the numbers of loci $\left(k b_{1}\right.$ and $\left.k b_{2}\right)$ that segregate in the single crosses and on the background loci ( $k f$ ) that have different alleles fixed in each pair of parents. In practice, the statistics and distributions of $B, D$ and $D c$ samples differ significantly from each other for most of the characters. The statistics of the $D c$ sample, on the other hand, have values that often lie between those of $B$ and $D$ samples. In fact the statistical properties of the $D c$ sample are adequately mimicked by the joint distribution of the $B+D$ inbreds except for range and intertrait correlations.

Further, the extreme recombinant inbreds from the double cross sample are not generally superior to those from the $B$ and $D$ samples. However, they do cover between 88 per cent to 109 per cent of the total range that is jointly covered by the extreme inbreds of the $B$ and $D$ samples. Theoretically the double cross is a better source when almost all of the genetic variability is contributed by the background loci $(k f)$ and the alleles are dispersed between the two pairs of parents.

\section{INTRODUCTION}

In our previous papers (Jinks and Pooni, 1976; Pooni and Jinks, 1978; 1985) we have described procedures for predicting the phenotypic distributions of inbred lines that can be extracted from the crosses between two, three and four pure breeding parents. The reliability of these procedures under field conditions has also been demonstrated by comparing the predicted and observed proportions of random inbred families that were extracted from the $F_{2}$ generations of $V_{1} \times V_{5}$ and $V_{2} \times V_{12}$ crosses of Nicotiana rustica (Jinks and Pooni, 1976; 1980; Pooni, Jinks and Pooni, 1980) and from the double cross $\left(V_{1} \times V_{5}\right) \times\left(V_{2} \times V_{12}\right)$ (Pooni and Jinks, 1985; Pooni, Jinks and Yohannes, 1985). While the relative potentials of the single and double crosses have been predicted and compared on a number of occasions the respective phenotypic distributions of the inbred lines have not been compared. In the present paper we compare the three sets of inbred lines for their expected and observed distributions. Theoretically, we obtain the expectations of various statistics that are associated with their phenotypic distributions using the models developed by Eberhart (1964) and Pooni and Jinks (1985). In practice we compare the same statistics by analysing the data recorded on these inbreds during the summer of 1983.

\section{THEORETICAL EXPECTATIONS}

\section{(i) Model}

Theoretically we shall derive the genetical expectations of various statistics such as overall mean, variance, skewness and kurtosis of each group of inbred lines and compare them under four genetical situations that can be defined by the absence and presence of non-allelic interactions and linkage disequilibrium. They are defined as following:

(a) Simple inheritance with no epistasis and linkage equilibrium (model 1);

(b) No epistasis but linkage disequilibrium (model 2); 

3);

(c) Epistasis with linkage equilibrium (model

(d) Epistasis with linkage disequilibrium (model 4).

Further, we shall follow Pooni and Jinks (1985) and divide the " $k$ " loci that segregate in a double cross into those incorporating two $\left(k^{\text {II }}\right)$, three $\left(k^{\text {III }}\right)$ and four $\left(k^{\mathrm{IV}}\right)$ alleles respectively and subdivide the $k^{\text {II }}$ group into:

(1) loci $\left(k b_{1}\right)$ that segregate in $P_{1} \times P_{2}$ cross but not in $P_{3} \times P_{4}$ cross;

(2) loci $\left(k b_{2}\right)$ that segregate in $P_{3} \times P_{4}$ cross but not in $P_{1} \times P_{2}$ cross;

(3) loci $(k b)$ that segregate both in $P_{1} \times P_{2}$ and $P_{3} \times P_{4}$ crosses and

(4) loci $(k f)$ that segregate in the double cross but have alternative alleles fixed in $P_{1} \times P_{2}$ and $P_{3} \times P_{4}$ crosses.

Here $k b+k b_{1}+k b_{2}+k f=k^{\mathrm{II}}$ and $k b_{1}=$ $k_{1}^{\mathrm{II}}+k_{2}^{\mathrm{II}}, k b_{2}=k_{3}^{\mathrm{II}}+k_{4}^{\mathrm{II}}, k b=k_{6}^{\mathrm{II}}+k_{7}^{\mathrm{II}}$ and $k f=k_{5}^{\mathrm{II}}$ (see Pooni and Jinks (1985) for definitions of $k_{1}^{\mathrm{II}}, \ldots, k_{7}^{\mathrm{II}}$, etc.).

\section{(ii) Overall means}

For a simple case of no epistasis and linkage equilibrium (model 1) and two alleles per locus the overall means of the populations have the following expectations:

$$
\begin{aligned}
\bar{F}_{\infty}(1 \times 2) & =m^{\prime}+\sum_{i=1}^{k b_{2}} d_{i}+\sum_{i=1}^{k f} d_{i} \\
& =m^{\prime}+[d] k b_{2}+[d] k f ; \\
\bar{F}_{\infty}(3 \times 4) & =m^{\prime}+\sum_{i=1}^{k b_{1}} d_{i}-\sum_{i=1}^{k f} d_{i} \\
& =m^{\prime}+[d] k b_{1}-[d] k f ; \\
\bar{D} c_{1} S_{\infty}(1 \times 2) \times(3 \times 4) & =m^{\prime}+\frac{1}{2} \sum_{i=1}^{k b_{1}} d_{i}+\frac{1}{2} \sum_{i=1}^{k b_{2}} d_{i} \\
& =m^{\prime}+\frac{1}{2}[d] k b_{1}+\frac{1}{2}[d] k b_{2} .
\end{aligned}
$$

While it is clear that $\bar{F}_{\infty}(1 \times 2)$ and $\bar{F}_{\infty}(3 \times 4)$ can differ because $[d] k b_{1} \neq[d] k b_{2}$, or $[d] k f \neq 0$ and $[d] k b_{2}+[d] k f \neq[d] k b_{1}-[d] k f$; the overall mean $\bar{D} c_{1} S_{\infty}(1 \times 2) \times(3 \times 4)$ will however fall between $\bar{F}_{\infty}(1 \times 2)$ and $\bar{F}_{\infty}(3 \times 4)$. These expectations remain the same for model 2 but are modified as:

$$
\begin{aligned}
& \bar{F}_{\infty}(1 \times 2)=(\text { ii. } 1)+[i] k b_{2}+[i] k f+[i] k b_{2} k f ; \\
& \bar{F}_{\infty}(3 \times 4)=(\text { ii. } 2)+[i] k b_{1}+[i] k f-[i] k b_{1} k f
\end{aligned}
$$

and

$$
\begin{aligned}
& \bar{D} c_{1} S_{\infty}(1 \times 2) \times(3 \times 4) \\
& \quad=(\mathrm{ii} .3)+\frac{1}{4}[i] k b_{1}+\frac{1}{4}[i] k b_{2}+\frac{1}{4}[i] k b_{1} k b_{2} .
\end{aligned}
$$

when epistasis is significant (model 3). Here [i] $k b_{1}$ etc. represent the additive $\times$ additive interaction effects of various groups of loci and are defined as:

$$
\begin{array}{ll}
{[i] k b_{1}=\sum_{i=1, j=i+1}^{k b_{1}} i_{i j},} & {[i] k b_{2}=\sum_{i=1, j=i+1}^{k b_{2}} i_{i j} ;} \\
{[i] k b_{1} k f=\sum_{i=1}^{k b_{1}} \sum_{j=1}^{k f} i_{i j} ;} & {[i] k b_{2} k f=\sum_{i=1}^{k b_{2}} \sum_{j=1}^{k f} i_{i j} ;}
\end{array}
$$

and $[i] k b_{1} k b_{2}=\sum_{i=1}^{k b_{1}} \sum_{j=1}^{k b_{2}} i_{i j}$ (see Mather and Jinks (1982) for notations).

In the presence of non-allelic interactions and linkage disequilibrium (model 4) the expected means become:

$$
\begin{gathered}
\bar{F}_{\infty}(1 \times 2)=(\mathrm{ii} .4)_{-R}^{+C} \sum_{i=1, j=i+1}^{k b+k b_{1}} \frac{\left(1-2 p_{i j}\right)}{\left(1+2 p_{i j}\right)} i_{i j} ; \\
\bar{F}_{\infty}(3 \times 4)=(\mathrm{ii} .5)_{-R}^{+C} \sum_{i=1, j=i+1}^{k b+k b_{2}} \frac{\left(1-2 p_{i j}\right)}{\left(1+2 p_{i j}\right)} i_{i j} ; \\
\bar{D} c_{1} S_{\infty}(1 \times 2) \times(3 \times 4) \\
=(\mathrm{ii} .3)_{-R}^{+C} \sum_{i=1, j=i+1}^{k b_{1}+k b_{2}} \frac{\left(1-p_{i j}\right)}{\left(1+p_{i j}\right)} i_{i j} \\
\quad+C \sum_{-R}^{k b+k f} i_{i=1, j=i+1}^{i_{i j}+C} \sum_{i=1}^{k b_{1}+k b_{2}} \sum_{j=1}^{k b+k f} \frac{1-2 p_{i j}}{2\left(1+2 p_{i j}\right)} i_{i j} ;
\end{gathered}
$$

respectively, where $p_{i j}$ is the frequency of recombination between the $i$ th and $j$ th locus of the respective group of loci. It is apparent that the relationships established earlier for the model 1 and model 2 may not apply to the overall means under model 3 and model 4.

\section{(iii) Additive genetic variances}

The corresponding genetic variances of the three groups of inbred lines have the expectations:

$$
\begin{aligned}
& \sigma^{2} F_{\infty}(1 \times 2)=\sum_{i=1}^{k b_{1}} d_{i}^{2}+\sum_{i=1}^{k b} d_{i}^{2}=V d k b_{1}+V d k b ; \\
& \sigma^{2} F_{\infty}(3 \times 4)=\sum_{i=1}^{k b_{2}} d_{i}^{2}+\sum_{i=1}^{k b} d_{i}^{2}=V d k b_{2}+V d k b ;
\end{aligned}
$$


and

$$
\begin{aligned}
\sigma^{2} D c_{1} & S_{\infty}(1 \times 2) \times(3 \times 4) \\
= & \frac{3}{4} \sum_{i=1}^{k b_{1}} d_{i}^{2}+\frac{3}{4} \sum_{i=1}^{k b_{2}} d_{i}^{2}+\sum_{i=1}^{k b} d_{i}^{2}+\sum_{i=1}^{k f} d_{i}^{2} \\
= & \frac{3}{4} V d k b_{1}+\frac{3}{4} V d k b_{2}+V d k b+V d k f
\end{aligned}
$$

for model 1 and

$$
\begin{aligned}
& \sigma^{2} F_{\infty}(1 \times 2)=(\mathrm{iii} .1)_{-R}^{+C} \sum_{i=1, j=i+1}^{k b_{1}+k b} \frac{2\left(1-2 p_{i j}\right)}{\left(1+2 p_{i j}\right)} d_{i} d_{j} ; \\
& \sigma^{2} F_{\infty}(3 \times 4)=(\text { iii. } 2)_{-R}^{+C} \sum_{i=1, j=i+1}^{k b_{2}+k b} \frac{2\left(1-2 p_{i j}\right)}{\left(1+2 p_{i j}\right)} d_{i} d_{j},
\end{aligned}
$$

and

$$
\begin{aligned}
\sigma^{2} D c_{1} & S_{\infty}(1 \times 2) \times(3 \times 4) \\
= & (\mathrm{iii} .3)_{-R}^{+C} \sum_{i=1, j=i+1}^{k b_{1}+k b_{2}} \frac{3\left(1-2 p_{i j}\right)}{2\left(1+2 p_{i j}\right)} d_{i} d_{j} \\
& { }_{-R}^{+C} \sum_{i=1, j=i+1}^{k b+k f} \frac{2\left(1-2 p_{i j}\right)}{1+2 p_{i j}} d_{i} d_{j} \\
& +{ }_{-R}^{+C} \sum_{i=1}^{k b_{1}+k b_{2}} \sum_{j=1}^{k b+k f} \frac{\left(1-2 p_{i j}\right)}{\left(1+2 p_{i j}\right)} d_{i} d_{j}
\end{aligned}
$$

for model 2. In the presence of non-allelic interactions (model 3)

$$
\begin{gathered}
\sigma^{2} F_{\infty}(1 \times 2)=(\mathrm{iii} .1)+\sum_{i=1, j=i+1}^{k b_{1}+k b} i_{i j}^{2} ; \\
\sigma^{2} f_{\infty}(3 \times 4)=(\mathrm{iii} .2)+\sum_{i=1, j=i+1}^{k b_{2}+k b} i_{i j}^{2},
\end{gathered}
$$

and

$$
\begin{aligned}
& \sigma^{2} D c_{1} S_{\infty}(1 \times 2)(3 \times 4) \\
&=(i i i .3)+\sum_{i=1, j=i+1}^{k b_{1}+k b_{2}+k b+k f} i_{i j}^{2} \\
&-\frac{1}{16} \sum_{i=1, j=i+1}^{k b_{1}+k b_{2}} i_{i j}^{2+(++-)^{3 / 4}} \sum_{i=1, j=i+1}^{k b_{1}+k b_{2}}\left[\left(d_{i-R}^{+C} d_{j}\right) i_{i j}\right] \\
&+\left[\sum_{i=1}^{k b_{1}+k b_{2}} \sum_{j=1}^{k b+k f} d_{i} i_{i j}\right]+\frac{3}{8} \sum_{i=1, j=i+1, s=j+1}^{k b_{1}+k b_{2}} i_{i j} i_{i s}
\end{aligned}
$$

Here ++ and -- stand for association for increasing and decreasing alleles respectively.

These expectations are further modified to:

$$
\sigma^{2} F_{\infty}(1 \times 2)=(\mathrm{iii} .4)+\sum_{i=1, j=i+1}^{k b_{1}+k b} \frac{8 p_{i j}}{\left(1+2 p_{i j}\right)^{2}} i_{i j}^{2}
$$

$$
\sigma^{2} F_{\infty}(3 \times 4)=(\mathrm{iii} .5)+\sum_{i=1, j=i+1}^{k b_{2}+k b} \frac{8 p_{i j}}{\left(1+2 p_{i j}\right)^{2}} i_{i j}^{2} ;
$$

and

$$
\begin{aligned}
\sigma^{2} D c_{1} & S_{\infty}(1 \times 2) \times(3 \times 4) \\
= & (i i i .6)+\sum_{i=1, j=i+1}^{k b_{1}+k b_{2}} \frac{8 p_{i j}}{\left(1+2 p_{i j}\right)^{2}} i_{i j}^{2} \\
& -\sum_{i=1, j=i+1}^{k b_{1}+k b_{2}} \frac{p_{i j}\left(2-3 p_{i j}\right)}{\left(1+2 p_{i j}\right)^{2}} i_{i j}^{2} \\
& +(++)\left[\sum_{i=1, j=i+1}^{k b_{1}+k b_{2}} \frac{3 p_{i j}}{\left(1+2 p_{i j}\right)}\left(d_{i-R}^{+C} d_{j}\right) i_{i j}\right] \\
& -(--)) \\
& +\left[\sum_{i=1}^{k b_{1}+k b_{2}} \sum_{j=1}^{k b+k f} d_{i} i_{i j}\right]+\text { cross products of } i \text { 's }
\end{aligned}
$$

in the presence of epistasis and linkage disequilibrium (model 4).

\section{(iv) Non-normality}

The expectations and effects of non-normality on the distributions of inbred lines that can be extracted from crosses between two pure breeding varieties have been described by Jinks and Pooni (1981) and Pooni, Jinks and Cornish (1977). While the distributons of $F_{\infty}(1 \times 2)$ and $F_{\infty}(3 \times 4)$ populations are symmetrical with a coefficient of skewness $\left(g_{1}\right)$ equal to zero both in the absence and presence of a linkage disequilibrium (models 1 and 2) they become asymmetric $\left(g_{1} \neq 0\right)$ in the presence of non-allelic interactions. For model 3,

$$
\begin{aligned}
& \text { skewness } F_{\infty}(1 \times 2)=\left[6 \sum_{i=1, j=i+1}^{k b_{1}+k b} d_{i} d_{j} i_{i j}\right. \\
& \left.+6 \sum_{i=1, j=i+1, s=j+1}^{k b_{1}+k b} i_{i j} i_{i} i_{j s}\right] /(\text { Variance in iii. } 7)^{3 / 2}
\end{aligned}
$$

skewness $F_{\infty}(3 \times 4)=\left[6 \underset{i=1, j=i+1}{k b_{2}+k b} d_{i} d_{j} i_{i j}\right.$

$$
\left.+6 \sum_{i=1, j=i+1, s=j+1}^{k b_{2}+k b} i_{i j} i_{i s} i_{j s}\right] /(\text { Variance in iii. } 8)^{3 / 2}
$$

(see Pooni et al., 1977 for definitions) and for 
model 4 these expectations become,

$$
\begin{aligned}
& \text { skewness } F_{\infty}(1 \times 2)=\left[24 \sum_{i=1, j=i+1}^{k b_{1}+k b} \frac{p_{i j}}{\left(1+2 p_{i j}\right)} d_{i} d_{j} i_{i j}\right. \\
& +384 \sum_{i=1, k=i+1, s=j+1}^{k b_{1}+k b} \\
& \left.\frac{p_{i j} \cdot p_{i s} \cdot p_{j s}}{\left(1+2 p_{i j}\right)\left(1+2 p_{i s}\right)\left(1+2 p_{j s}\right)} i_{i j} i_{i s} i_{j s}\right] \\
& /(\text { Variance in iii. } 10)^{3 / 2}
\end{aligned}
$$

and

skewness $F_{\infty}(3 \times 4)$

$$
\begin{aligned}
= & {\left[24 \sum_{i=1, j=i+1}^{k b_{2}+k b} \frac{p_{i j}}{\left(1+2 p_{i j}\right)} d_{i} d_{j} i_{i j}\right.} \\
& +384 \sum_{i=1, j=i+1, s=j+1}^{k b_{2}+k b} \\
& \left.\frac{p_{i j} \cdot p_{i s} \cdot p_{j s}}{\left(1+2 p_{i j}\right)\left(1+2 p_{i s}\right)\left(1+2 p_{j s}\right)} i_{i j} i_{i s} i_{j s}\right] \\
& /(\text { Variance in iii.11 })^{3 / 2} .
\end{aligned}
$$

Here, the parameters $p_{i j}, p_{i s}, p_{j s}, i_{i j}, i_{i s}$ and $i_{j s}$ are the coefficients of recombination and additive $x$ additive interactioins of the $i$ th and $j$ th, $i$ th and $s$ th, and $j$ th and sth pairs of loci.

The corresponding coefficients of skewness for inbreds derived from the double cross have rather complex expectations. For models 1 and 2

skewness $D c_{1} S_{\infty}(1 \times 2) \times(3 \times 4)=$

$$
\mp \frac{3}{4}\left[\sum_{i=1}^{k b_{1}+k b_{2}} d_{i}^{3}\right] /(\text { variances in iii.3 or iii. } 6)^{3 / 2}
$$

and for model 3 it takes a value which is equal to:

$$
\begin{aligned}
& \left\{ \pm \frac{3}{4}\left[\sum_{i=1}^{k b_{1}+k b_{2}} d_{i}^{3}\right] \pm \frac{11}{32} \sum_{i=1, j=i+1}^{k b_{1}+k b_{2}} i_{i j}^{3}\right] \pm \\
& \pm \frac{9}{8}\left[\sum_{i=1, j=i+1}^{k b_{1}+k b_{2}}\left(d_{i} \pm d_{j}\right) i_{i j}^{2}\right] \pm-\left[\sum_{i=1, j=i+1}^{k b_{1}+k b_{2}}\left(d_{i}^{2}+d_{j}^{2}\right) i_{i j}\right] \\
& \quad+\frac{27}{8}\left[\sum_{i=1, j=i+1}^{k b_{1}+k b_{2}} d_{i} d_{j} i_{i j}\right]+6\left[\sum_{i=1, j=i+1}^{k b+k f} d_{i} d_{j} i_{i j}\right] \\
& \left.+6\left[\sum_{i=1, j=i+1, s=j+1}^{k b+k f} i_{i j} i_{i s} i_{j s}\right]+\text { cross products }\right\} \\
& \\
& \quad /(\text { Variance in iii. } 9)^{3 / 2} .
\end{aligned}
$$

The expected contributions of these and other components that contribute to the skewness in the presence of epistasis and linkage disequilibrium (model 4) are further complicated by the imbalances between the parental and recombinant types of gametes. In general repulsion linkages are expected to reduce skewness and coupling linkages increase it. However, the overall asymmetry of the inbred lines derived from a double cross will be largely determined by the overall inequality of allelic frequencies that remains after internal cancellations between the gene frequencies at various loci.

The expected kurtosis for the $F_{\infty}(1 \times 2)$ and $F_{\infty}(3 \times 4)$ populations under model 1 situations is equal to:

$$
\left[\left(\sum_{i=1}^{k b_{1}+k b} d_{i}^{4}+6 \sum_{i=1, j=i+1}^{k b_{1}+k b} d_{i}^{2} d_{j}^{2}\right) /\left(\sum_{i=1}^{k b_{1}+k b} d_{i}^{2}\right)^{2}\right]-3
$$

and

$$
\left[\left(\sum_{i=1}^{k b_{2}+k b} d_{i}^{4}+6 \sum_{i=1, j=i+1}^{k b_{2}+k b} d_{i}^{2} d_{j}^{2}\right) /\left(\sum_{i=1}^{k b_{2}+k b} d_{i}^{2}\right)^{2}\right]-3,
$$

respectively.

The corresponding expected kurtosis for the $D c_{1} S_{\infty}(1 \times 2) \times(3 \times 4)$ population is:

$$
\begin{gathered}
{\left[\left(\sum_{i=1}^{k b+k f} d_{i}^{4}+6 \sum_{i=1}^{k b+k f} d_{i}^{2} d_{j}^{2}+\frac{21}{16} \sum_{i=1}^{k b_{1}+k b_{2}} d_{i}^{4}\right.\right.} \\
\left.\quad+\frac{27}{8} \sum_{i=1, j=i+1}^{k b_{1}+k b_{2}} d_{i}^{2} d_{j}^{2}+\frac{9}{8} \sum_{i=1}^{k b_{1}+k b_{2} k b+k f} \sum_{j=1}^{k} d_{i}^{2} d_{j}^{2}\right) \\
\left./\left(\sum_{i=1}^{k b+k f} d_{i}^{2}+\frac{3}{4} \sum_{i=1}^{k b_{1}+k b_{2}} d_{i}^{2}\right)^{2}\right]-3
\end{gathered}
$$

Both epistasis and linkage disequilibrium affect the magnitude of $g_{2}$. In general, epistasis produces positive kurtosis because it increases the frequencies of some phenotypes and reduces others. Linkage disequilibrium, on the other hand, makes the distribution flat when the linkages are predominantly in the coupling phase and increases the peakedness when they are predominantly in the repulsion phase.

\section{(v) Extreme Inbreds}

Jinks and Perkins (1972) have defined the range of inbred lines that can be extracted from a cross between two pure breeding varieties as $m+\sum_{i=1}^{k} d_{i}$ and $m-\sum_{i=1}^{k} d_{i}$ in the absence of epistasis and $m+\sum_{i=1}^{k} d_{i}+\sum_{i=1, j=i+1}^{k} i_{i j}$ and $m-\sum_{i=1}^{k} d_{i}+$ $\sum_{i=1, j=i+1}^{k} i_{i j}$ when nonallelic interactions are present. On this model the highest and lowest scoring 
inbreds that can be exacted from the $1 \times 2$ and $3 \times 4$ crosses through inbreeding have the expected scores of:

$$
\begin{aligned}
m^{\prime}+ & {\left[\sum_{i=1}^{k b_{2}+k f} d_{i}\right]+\sum_{i=1}^{k b_{1}+k b} d_{i} ; \quad m^{\prime}+\left[\sum_{i=1}^{k b_{2}+k f} d_{i}\right] } \\
& -\sum_{i=1}^{k b_{1}+k b} d_{i} ; \\
m^{\prime}+ & {\left[\sum_{i=1}^{k b_{1}+k f} d_{i}\right]+\sum_{i=1}^{k b_{2}+k b} d_{i} ; \text { and } } \\
m^{\prime}+ & {\left[\sum_{i=1}^{k b_{1}+k f} d_{i}\right]-\sum_{i=1}^{k b_{2}+k b} d_{i} ; }
\end{aligned}
$$

for models 1 and 2 and

$$
\begin{aligned}
& m^{\prime}+\left[\sum_{i=1}^{k b_{2}+k f} d_{i}\right]+\sum_{i=1}^{k b_{1}+k b} d_{i}+\left[\sum_{i=1, j=i+1}^{k b_{2}+k f} i_{i j}\right] \\
& +\sum_{i=1, j=i+1}^{k b_{1}+k b} i_{i j}+\left[\sum_{i=1}^{k b_{2}+k f} \sum_{j=1}^{k b_{1}+k b} i_{i j}\right] ; \\
& m^{\prime}+\left[\sum_{i=1}^{k b_{2}+k f} d_{i}\right]-\sum_{i=1}^{k b_{1}+k b} d_{i}+\left[\sum_{i=1, j=i+1}^{k b_{2}+k f} i_{i j}\right] \\
& +\sum_{i=1, j=i+1}^{k b_{1}+k b} i_{i j}-\left[\sum_{i=1}^{k b_{2}+k f} \sum_{j=1}^{k b_{1}+k b} i_{i j}\right] ; \\
& m^{\prime}+\left[\sum_{i=1}^{k b_{1}+k f} d_{i}\right]+\sum_{i=1}^{k b_{2}+k b} d_{i}+\left[\begin{array}{c}
k b_{1}+k f \\
i=1, j=i+1
\end{array}\right] \\
& +\sum_{i=1, j=i+1}^{k b_{2}+k b} i_{i j}+\left[\sum_{i=1}^{k b_{1}+k f} \sum_{j=1}^{k b_{2}+k b} i_{i j}\right]
\end{aligned}
$$

and

$$
\begin{aligned}
m^{\prime} & +\left[\sum_{i=1}^{k b_{1}+k f} d_{i}\right]-\sum_{i=1}^{k b_{2}+k b} d_{i}+\left[\sum_{i=1, j=i+1}^{k b_{1}+k f} i_{i j}\right] \\
& +\sum_{i=1, j=i+1}^{k b_{2}+k b} i_{i j}-\left[\sum_{i=1}^{k b_{1}+k f} \sum_{j=1}^{k b_{2}+k b} i_{i j}\right]
\end{aligned}
$$

for models 3 and 4. The components in [ ] are subjected to internal cancellations that are dependent on the degree of gene association between each pair of parents.

The corresponding highest and lowest scoring inbreds that can be extracted from the double cross $(1 \times 2) \times(3 \times 4)$ have the expectations of $m^{\prime}+$ $\sum_{i=1}^{k b_{1}+k b_{2}+k b+k f} d_{i}$ and $m^{\prime}-\sum_{i=1}^{k b_{1}+k b_{2}+k b+k f} d_{i}$ for models 1 and 2 , and $m^{\prime}+\sum_{i=1}^{k b_{1}+k b_{2}+k b+k f} d_{i}+$ $\sum_{\substack{k=1, j=i+1 \\ k b_{1}+k b_{2}+k b+k f}} i_{i j}$ and $m^{\prime}-\sum_{i=1}^{k b_{1}+k b_{2}+k f+k f} d_{i}+$

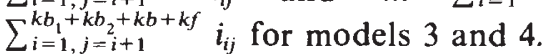

\section{MATERIAL}

The materials consists of:

(a) 82 random inbred lines ( $B$ lines) extracted from the $\mathrm{F}_{2}$ of $V 1 \times V 5$ cross of Nicotiana rustica.

(b) 60 random inbreds ( $D$ lines) extracted from the $F_{2}$ of $V 2 \times V 12$ cross of $N$. Rustica.

(c) 120 random inbreds ( $D c$ lines) extracted from the double cross of $V 1 \times V 5$ and $V 2 \times V 12$.

Each set of inbreds was produced by the method of single seed descent and are $F_{17}, F_{14}$ and $D c_{1} S 6$ generation of selfing, respectively. Ten plants from each of these 262 families were raised during summer 1983 as a part of a much larger experiment, (see Pooni, Jinks and Yohannes, 1985 for details) and each of the 2620 plants were individually scored for the following characters:

H4 Height in $\mathrm{cm} 4$ weeks after planting in the field

H6 Height in $\mathrm{cm} 6$ weeks after planting in the field

FT Flowering time in days from 1st July

$H F T$ Height in $\mathrm{cm}$ at the time of flowering

$L L \quad$ Leaf length in $\mathrm{cm}$ of the largest leaf blade

$L W \quad$ Leaf width in $\mathrm{cm}$ of the largest leaf blade

FH Final plant height in $\mathrm{cm}$ at the end of season.

Because of poor germination and damage during the growing season no data could be collected on 50 plants and only four characters were scored on two others. Nineteen of these 52 plants belonged to $B 10$ and $D 32$ inbreds and these genotypes were excluded from the respective samples. Consequently the $B$ and $D$ samples were reduced to 81 and 59 inbreds only.

The data were processed through the University's Multics Computer to obtain and compare the etimates of various statistics. In addition we also analysed $59 \mathrm{~B}$ and $59 \mathrm{D}$ inbreds as a combined $B+D$ sample and compared it with the $D c$ sample to test if the latter is more variable than the former.

\section{RESULTS AND DISCUSSION}

\section{(i) Comparisons of statistics obtainable from family means}

Estimates of the overall mean, additive genetic variance, skewness $\left(g_{1}\right)$ and kurtosis $\left(g_{2}\right)$ for the $B, D, B+D$ and $D c$ samples and the results of their pairwise comparisons for each character are tabulated in tables 1,2 and 3 , respectively. The 
H. S. POONI, J. L. JINKS AND D. YOHANNES

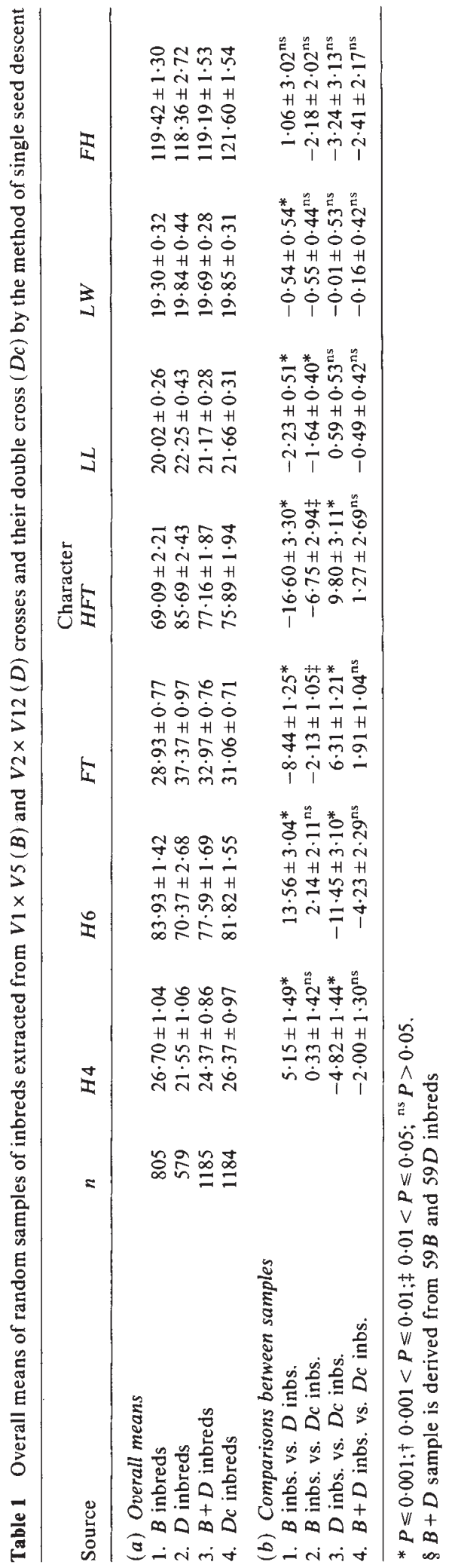


Table 2 Estimates of additive genetic variances of $B, D, B+D$ and $D c$ inbred lines extracted from $V 1 \times V 5$ and $V 2 \times V 12$ crosses and their double cross, and the tests of homogeneity of these components between pairs of samples

\begin{tabular}{|c|c|c|c|c|c|c|c|c|}
\hline Source & $\mathrm{df}$ & $H 4$ & $H 6$ & $F T$ & $\begin{array}{l}\text { Character } \\
H F T\end{array}$ & $L L$ & $L W$ & $F H$ \\
\hline \multicolumn{9}{|c|}{ (a) Additive genetic component } \\
\hline 2. $D$ inbreds & 58 & $62 \cdot 73$ & $407 \cdot 48$ & $53 \cdot 70$ & $329 \cdot 15$ & $10 \cdot 44$ & $10 \cdot 46$ & $414 \cdot 48$ \\
\hline 3. $B+D$ inbreds & 117 & $84 \cdot 45$ & $323 \cdot 87$ & $67 \cdot 29$ & $396 \cdot 48$ & 8.77 & 8.96 & $262 \cdot 75$ \\
\hline 4. $D c$ inbreds & 119 & $108 \cdot 14$ & $272 \cdot 12$ & $58 \cdot 13$ & $427 \cdot 44$ & $10 \cdot 64$ & $10 \cdot 47$ & $268 \cdot 14$ \\
\hline \multicolumn{9}{|c|}{ (b) Comparisons between samples } \\
\hline 1. $B$ inbs vs, $D$ inbs & & $1 \cdot 23^{\text {ns }}$ & $15 \cdot 19 * \S$ & $0 \cdot 34^{\mathrm{ns}}$ & $0 \cdot 33^{\text {ns }}$ & $7 \cdot 41 \dagger$ & $1 \cdot 49^{\text {ns }}$ & $21 \cdot 29 *$ \\
\hline 2. $B$ inbs vs. $D c$ inbs & & $1 \cdot 40^{\text {ns }}$ & $6 \cdot 33 \ddagger$ & $1 \cdot 08^{\text {ns }}$ & $0 \cdot 30^{\text {ns }}$ & $8.87 \dagger$ & $2 \cdot 09^{\text {ns }}$ & $9.97^{*}$ \\
\hline 3. $D$ inbs vs. $D c$ inbs & & $4 \cdot 38^{*}$ & $3 \cdot 33^{\text {ns }}$ & $0 \cdot 11^{\mathrm{ns}}$ & $1 \cdot 13^{\text {ns }}$ & $0.01^{\text {ns }}$ & $0 \cdot 00^{\text {ns }}$ & $3 \cdot 82 \ddagger$ \\
\hline 4. $B+D$ inbs vs. $D c$ inbs & & $1.69^{\text {ns }}$ & $0.83^{\text {ns }}$ & $0.61^{\mathrm{ns}}$ & $0 \cdot 16^{\text {ns }}$ & $1 \cdot 02^{\text {ns }}$ & $0 \cdot 64^{\text {ns }}$ & $0.01^{\text {ns }}$ \\
\hline
\end{tabular}

$\S$ For probabilities see Table 1 .

Table 3 Coefficients of skewness and kurtosis of the family means for the $B, D, B+D$ and $D c$ samples of inbred lines extracted from the $V 1 \times V 5$ and $V 2 \times V 12$ crosses of Nicotiana rustica and their double cross

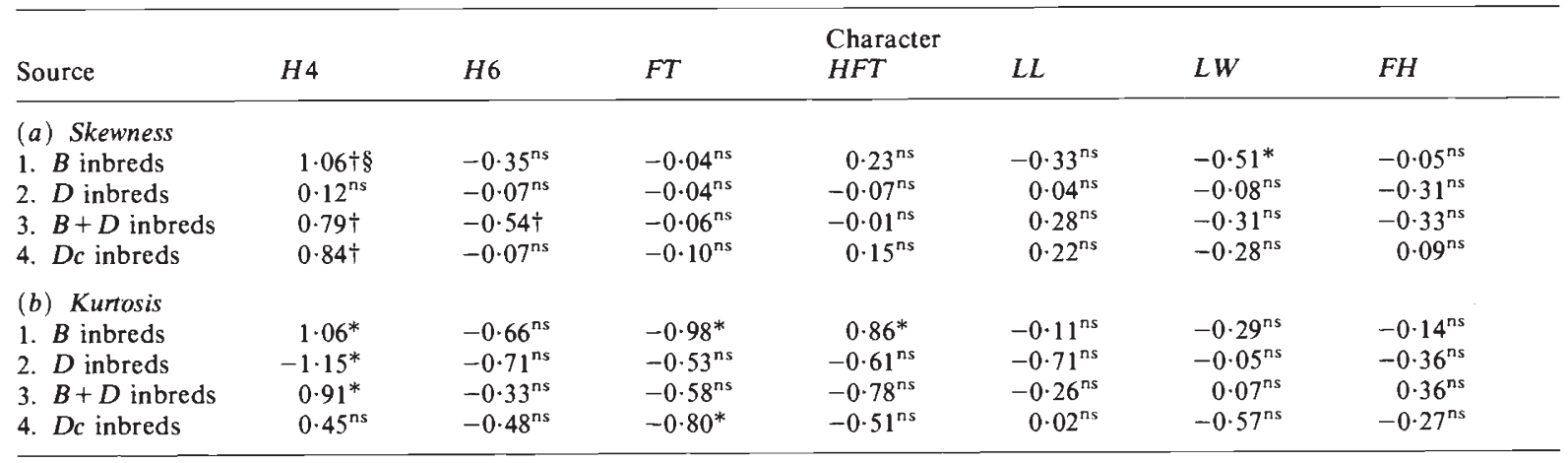

$\S$ All comparisons are non-significant except between $B$ and $D$ and $D$ and $D c$ for skewness and kurtosis of character $H 4$ For probabilities see table 1

overall means and the coefficients of skewness and kurtosis were estimated and compared by the conventional statistical manipulations of the family means (see Snedecor and Cochran, 1974 for procedures). The additive genetic variance, on the other hand, has been estimated from the between families and within families mean squares of each sample by the method of weighted least squares using the models of Jinks and Pooni (1984) and Pooni, Jinks and Yohannes (1985). These estimates were compared with each other by the methods of Pooni, Jinks and Pooni (1980).

It is clear from these results that the statistics of $B$ and $D$ samples often differ in magnitude. For instance the overall mean of the $B$ sample takes a larger value than that of the $D$ sample for $H 4, H 6$ and $F H$ and its value is smaller for $F T, H F T, L L$ and $L W$. These differences are highly significant for all characters except $L W$ and $F H$. The additive genetic variance of $D$ inbreds, on the other hand, is always larger than that of $B$ inbreds except for
$\mathrm{H} 4$ and the estimates differ significantly for three characters. Similarly, while the distribution of the $B$ sample shows significant skewness for $H 4$ and $L W$ and is negatively kurtosed for $H 4, F T$ and $H F T$, the $D$ inbreds are normally distributed for all characters except $H 4$. However, the two samples do not differ significantly for skewness or kurtosis for six of the seven characters under study.

The overall mean of the double cross $(D c)$ sample, on the other hand, differs significantly from that of $B$ or $D$ sample for five characters. But its value always falls within the range of $B$ and $D$ samples except for $L W$ and $F H$ for which it is the most vigorous. The $D c$ sample, nevertheless, has the largest additive genetic variance for $H 4, F T, H F T, L L$ and $L W$. However, it is significantly different from that of $B$ or $D$ sample for only $\mathrm{H} 4, \mathrm{H} 6$ and $\mathrm{FH}$. Further coefficients of skewness and kurtosis of the $D c$ sample are nonsignificant for five of the seven characters. 
Next we quantify the totality of these differences by comparing the phenotypic distributions of various samples. To make these comparisons we classify the family means of each sample into standardised class intervals that cover the whole range of the $B, D$ and $D c$ samples. Then we calculate the heterogeneity $\chi^{2}$ following Mather (1973). These $\chi^{2}$ are significant on ten occasions, i.e., five times for differences between $B$ and $D$ samples, four times for differences between $D$ and $D c$ samples and once for differences between $B$ and $D c$ samples. It is therefore obvious that the distributions of $B$ and $D c$ samples are very similar for most of the characters. The $\chi^{2}$ values, their significances and degrees of freedom are given for each character in table 4.
The statistics of the $D c$ sample, on the other hand, do not differ from those of $B+D$ sample for any of the 35 character/statistic combinations given in tables 1 to 4 .

A property of particular interest to the plant breeder is the capacity of a population to throw out extreme segregants. The three samples are, therefore, compared for the highest and lowest scores amongst their families and the results are given for each character in table 5. Again the $B$ and $D$ samples differ significantly. While the $B$ inbreds are extreme for the lowest score for $F T$, $H F T$ and $L L$ and for the highest score for $H 4$ the $D$ inbreds are extreme for the remaining character/category combinations. The lowest scores of $D c$ inbreds, on the other hand, are always inter-

Table 4 Values and significances of the contingency $\chi^{2}$ for the comparisons between the frequency distributions of $B$ and $D, B$ and $D c, D$ and $D c$ and $B+D$ and $D c$ samples of inbred lines for seven characters

\begin{tabular}{lccccccc}
\hline Comparison & & \multicolumn{3}{c}{ Character } & & \\
\cline { 5 - 7 } & $H 4$ & $H 6$ & $F T$ & $H F T$ & $L L$ & $L W$ & $F H$ \\
$B$ inbreds vs. $D$ inbreds & $6 \cdot 12^{*} \S(2)$ & $26 \cdot 75^{*}(4)$ & $25 \cdot 08^{*}(2)$ & $23 \cdot 20^{*}(4)$ & $6 \cdot 33^{*}(1)$ & $0 \cdot 44^{\text {ns }}(2)$ & $9 \cdot 18^{\text {ns }}(4)$ \\
$B$ inbreds vs. $D c$ inbreds & $4 \cdot 10^{\text {ns }}(4)$ & $5 \cdot 74^{\text {ns }}(4)$ & $6 \cdot 66^{\text {ns }}(4)$ & $6 \cdot 24^{\text {ns }}(5)$ & $3 \cdot 94^{*}(1)$ & $0 \cdot 87^{\text {ns }}(2)$ & $7 \cdot 32^{\text {ns }}(5)$ \\
$D$ inbreds vs. $D c$ inbreds & $11 \cdot 51 \dagger(3)$ & $29 \cdot 67^{*}(3)$ & $22 \cdot 06^{*}(3)$ & $9 \cdot 31^{\text {ns }}(5)$ & $6 \cdot 22^{*}(2)$ & $2 \cdot 28^{\text {ns }}(3)$ & $5 \cdot 46^{\text {ns }}(5)$ \\
$B+D$ inbreds vs. $D c$ inbreds & $6 \cdot 17^{\text {ns }}(4)$ & $10 \cdot 92^{\text {ns }}(5)$ & $5 \cdot 63^{\text {ns }}(5)$ & $0 \cdot 82^{\text {ns }}(7)$ & $0 \cdot 84^{\text {ns }}(2)$ & $3 \cdot 64^{\text {ns }}(3)$ & $5 \cdot 38^{\text {ns }}(5)$ \\
\hline
\end{tabular}

$\S$ For probabilities see table 1

Table 5 The minimum and maximum scores amongst the inbred lines of the $B, D, B+D$ and $D c$ samples and the tests of significance of differences between them

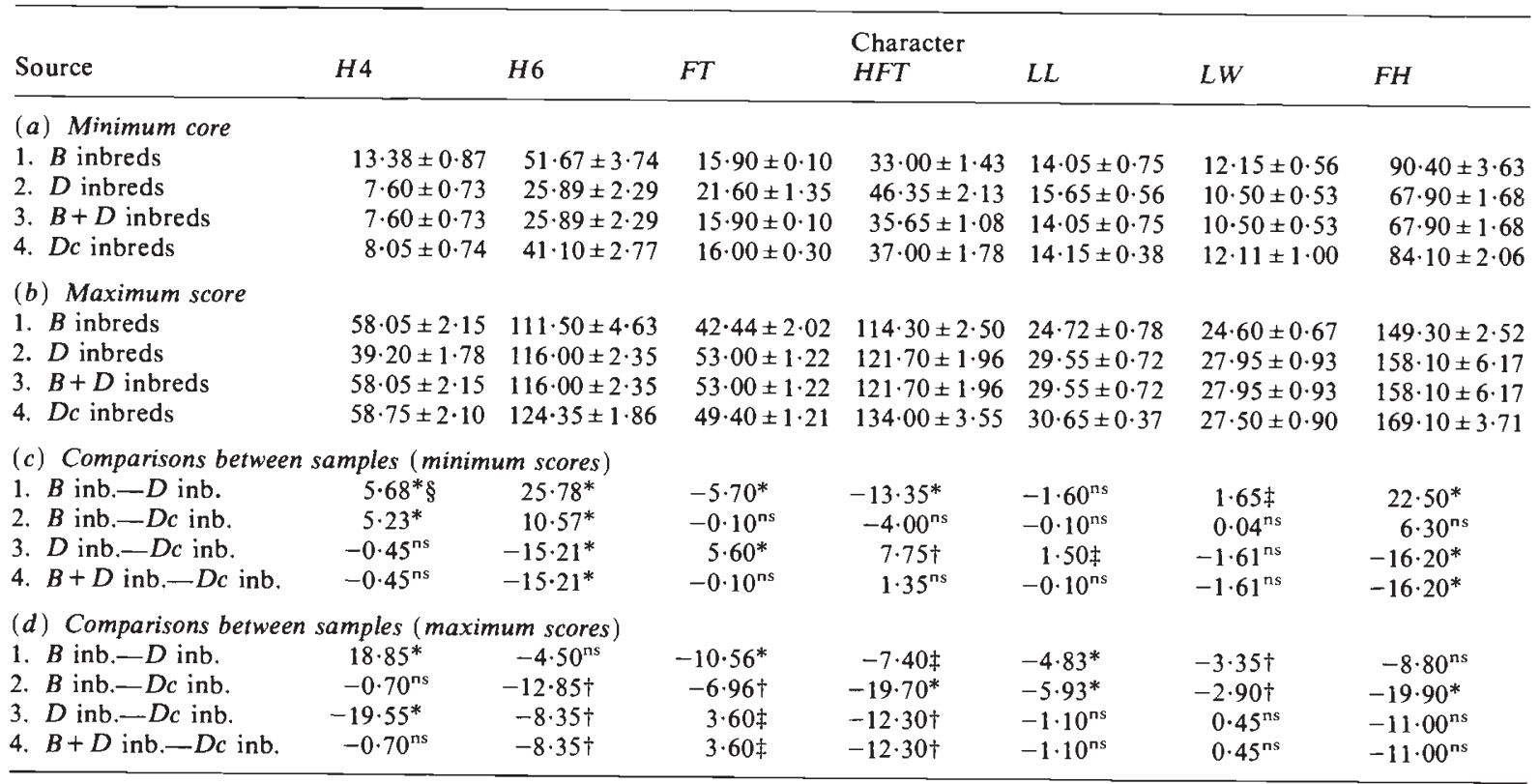

$\S$ Probabilities in table 1. 
mediate between those of $B$ and $D$ inbreds. But their scores are often closer to those for the $B$ sample and the two do not differ significantly for any character except $\mathrm{H} 4$ and $\mathrm{H} 6$.

The highest scores among the double cross inbreds are however also the highest amongst the three samples for all characters except $H F T$ and $L W$ and they are significantly larger on three occasions. Further, inbreds in the $D c$ sample also outperform those in the $B+D$ sample for a number of characters and these differences are significant for five characters.

Multiple crosses are often attempted to produce recombinants that combine good characteristics from more than two parents. They can be advantageous only when they provide more opportunities for obtaining a high degree of inter-trait improvement. We compare the inter-trait properties of the three samples by estimating the correlation coefficients for each of 21 pairs of characters. The genetic correlations, obtained from the genetic components of variances and covariances of each pair of characters, are tabulated for each of the $B$, $D, B+D$ and $D c$ samples in table 6 . While the magnitudes and directions of these correlations vary with the pairs of characters the $r$ values for
$B$ inbreds are generally larger (in absolute value) than those for $D$ inbreds. The correlation coefficients of $D c$ inbreds, on the other hand, take values that sometimes lie within and sometimes outside those of $B$ and $D$ inbreds but on certain occasions their $r$ values are smaller than those of $B$ and $D$ inbreds suggesting either additional recombination or reduction in linkage disequilibrium or both.

The $r$ values of $D c$ inbreds also differ considerably from those of $B+D$ inbreds. The two samples therefore must differ for the covariance component because their variances are very similar in magnitude.

The differences that we have observed between $B, D$ and $D c$ samples can be caused by the additive genetic deviations, the additive $\times$ additive interactions or both. The availability of six basic generations (parental varieties, $F_{1}, F_{2}$ and first backcrosses) from each of the two single crosses, four threeway crosses $\left(T c_{1}\right.$ 's), a double cross $\left(D c_{1}\right)$ and its first self $\left(D c_{1} S_{1}\right)$ allows us to test the significance of non-allelic interactions and thus determine the true nature of genetic differences that exist between samples. For the $B$ and $D$ samples non-allelic interactions were tested by Mather's (1949) $A, B$

Table 6 Genetic correlations amongst the random inbred lines of $B, D, B+D$ and $D c$ samples for 21 pairwise combinations of seven characters

\begin{tabular}{|c|c|c|c|c|c|c|c|}
\hline Character & Source & $H 6$ & $F T$ & $H F T$ & $L L$ & $L W$ & $F H$ \\
\hline$H 4$ & $\begin{array}{l}B \text { inbreds } \\
D \text { inbreds } \\
B+D \text { inbreds } \\
D c \text { inbreds }\end{array}$ & $\begin{array}{l}0 \cdot 79 \\
0 \cdot 87 \\
0 \cdot 81 \\
0 \cdot 78\end{array}$ & $\begin{array}{l}-0.83 \\
-0.81 \\
-0.83 \\
-0.83\end{array}$ & $\begin{array}{l}-0.56 \\
-0.10 \\
-0.45 \\
-0.52\end{array}$ & $\begin{array}{l}-0.72 \\
-0.42 \\
-0.60 \\
-0.51\end{array}$ & $\begin{array}{l}-0.69 \\
-0.31 \\
-0.55 \\
-0.51\end{array}$ & $\begin{array}{r}-0 \cdot 40 \\
0 \cdot 31 \\
0 \cdot 02 \\
-0 \cdot 15\end{array}$ \\
\hline$H 6$ & $\begin{array}{l}B \text { inbreds } \\
D \text { inbreds } \\
B+D \text { inbreds } \\
D c \text { inbreds }\end{array}$ & & $\begin{array}{l}-0.73 \\
-0.80 \\
-0.80 \\
-0.68\end{array}$ & $\begin{array}{l}-0.43 \\
+0.05 \\
-0.25 \\
-0.25\end{array}$ & $\begin{array}{l}-0.46 \\
-0.25 \\
-0.44 \\
-0.24\end{array}$ & $\begin{array}{l}-0 \cdot 37 \\
-0 \cdot 17 \\
-0 \cdot 27 \\
-0 \cdot 22\end{array}$ & $\begin{array}{l}0.01 \\
0.55 \\
0 \cdot 40 \\
0.28\end{array}$ \\
\hline$F T$ & $\begin{array}{l}B \text { inbreds } \\
D \text { inbreds } \\
B+D \text { inbreds } \\
D c \text { inbreds }\end{array}$ & & & $\begin{array}{l}0.91 \\
0.44 \\
0.73 \\
0.85\end{array}$ & $\begin{array}{l}0.70 \\
0.54 \\
0.66 \\
0.58\end{array}$ & $\begin{array}{l}0.61 \\
0.43 \\
0.49 \\
0.55\end{array}$ & $\begin{array}{r}0.61 \\
-0.07 \\
0.11 \\
0.42\end{array}$ \\
\hline$H F T$ & $\begin{array}{l}B \text { inbreds } \\
D \text { inbreds } \\
B+D \text { inbreds } \\
D c \text { inbreds }\end{array}$ & & & & $\begin{array}{l}0.60 \\
0.50 \\
0.58 \\
0.62\end{array}$ & $\begin{array}{l}0.51 \\
0.47 \\
0.46 \\
0.54\end{array}$ & $\begin{array}{l}0 \cdot 76 \\
0 \cdot 79 \\
0.64 \\
0.73\end{array}$ \\
\hline$L L$ & $\begin{array}{l}B \text { inbreds } \\
D \text { inbreds } \\
B+D \text { inbreds } \\
D c \text { inbreds }\end{array}$ & & & & & $\begin{array}{l}0.85 \\
0.84 \\
0.82 \\
0.79\end{array}$ & $\begin{array}{l}0.59 \\
0.41 \\
0.39 \\
0.56\end{array}$ \\
\hline$L W$ & $\begin{array}{l}B \text { inbreds } \\
D \text { inbreds } \\
B+D \text { inbreds } \\
D c \text { inbreds }\end{array}$ & & & & & & $\begin{array}{l}0.63 \\
0.41 \\
0.46 \\
0.55\end{array}$ \\
\hline
\end{tabular}


and $C$ scaling tests and Cavalli's (1952) joint scaling test. For the $D c$ inbreds epistasis was detected by testing the significance of $\left[4 \bar{D} c_{1}-\bar{T} c_{1}\{(V 1 \times\right.$ $V 5) \times V 2\}-\bar{T} c_{1}\{(V 1 \times V 5) \times V 12\}-\bar{T}_{1}\{(V 2 \times$ $\left.V 12) \times V 1\}-\bar{T} c_{1}\{(V 2 \times V 12) \times V 5\}\right]$ and $\left\{2 \bar{D} c_{1} S_{1}-\bar{D} c_{1}-\frac{1}{4}\left(\tilde{P}_{1}+\bar{P}_{3}+\bar{P}_{2}+\bar{P}_{4}\right)\right\}$ comparisons (see Pooni, Jinks and Yohannes, 1985 for symbols). The results are given in table 7 . It is clear that epistasis contributes significantly to the differences between various samples because non-allelic interactions are marginally significant for all characters.

\section{(ii) Comparisons of statistics obtainable from within family variances}

The families of $B$ and $D$ samples being highly inbred are expected to show no segregation. Thus the variation within each family is entirely environmental and in the absence of genotype $x$ micro-environmental interactions their averaged vaariance provides an estimate of the additive environmental variance $E_{w}$ (Pooni, Jinks and Jayasekara, 1978). However, the estimates of $E_{w}$ from the $B$ and $D$ inbreds will be the same only if the genotype $\times$ environmental interactions of their background loci $\left(k b_{1}\right.$ and $\left.k b_{2}\right)$ are also the same. Further, we expect the within family variances of each sample to be homogeneous and a significant heterogeneity between them is therefore evidence of differential genotypic sensitivity to the micro-environmental variation.

The families of the $D c$ sample, on the other hand, are not highly advanced inbreds. The variation within each family will therefore consist of a small residual heritable component and the additive environmental variance $E_{w}$. The two components, however, can be easily separated by the weighted least squares analysis using the models of Pooni, Jinks and Yohannes (1985). Further, family to family variation of the residual genetic component will also increase the heterogeneity of within family variances.

The estimates of $E_{w}$ and the $\chi_{(1)}^{2}$ tests of their homogeneity between samples, and Bartlett's tests of heterogeneity of within family variances for the $B, D, B+D$ and $D c$ samples are given in tables 8 and 9 , respectively. Reference to table 8 shows that the estimates of $E_{w}$ differ significantly between samples on at least 12 occasions. Therefore the three samples differ in the sensitivity of their background genotypes to the micro-environmental variation. Additive environmental variances of $D c$ and $B+D$ samples, however, are very similar in magnitude simply because the background loci are the same for both samples.
Bartlett's test of homogeneity of the within family variances shows significant heterogeneity within $B, D$ and $D c$ samples for all characters except $L L$ in respect of $B$ sample (table 9). There can be no doubt that genotype $\times$ micro-environmental interaction is responsible for this heterogeneity in $B$ and $D$ samples. This must be a major contributor in the $D c$ sample as well because the $\chi^{2}$ values of $D c$ and $B+D$ samples are very similar.

These tests, however, detect only the presence of genotype $\times$ micro-environmental interactions, and they do not indicate their cause. Such interactions can arise from the pleiotropic effects of genes that are segregating for mean performance or by segregation at independent loci that control environmental sensitivity. Furthermore the loci controlling mean performance and environmental sensitivity may or may not be in linkage equilibrium. Pleiotropy and linkage disequilibrium will however produce a linear relationship between the mean performance and environmental sensitivity that may differ between samples. Therefore, if a linear regression analysis is carried out for a sample using the family means as independent and the standard deviations ( $=\sqrt{\text { within family variance) }}$ as dependent variable, the regression mean square will indicate the degree of linear dependence of the additive genetic $\times$ micro-environmental interaction for that sample. Joint regression analysis for different pairs of samples will also provide the tests for the heterogeneity of the two regression coefficients.

The results of the regression analyses are given in table 10. In each case, the regression mean squares and their components have been tested against the corresponding remainder mean squares. Once again these results confirm the higherto well established pattern of differences between the samples. Altogether the tests of linear regression are significant on 18 occasions out of a total of 28 . Further the $B, D$ and $D c$ samples contrast with each other in that their regression coefficients are significantly different from each other for $H 6$ and FT. The regression coefficients of $D c$ and $B+D$ samples however do not differ significantly for any of the seven characters under study.

\section{CONCLUSIONS}

The main conclusion from section 2 is that the distributive properties of recombinant inbreds extracted from two single crosses and their double 


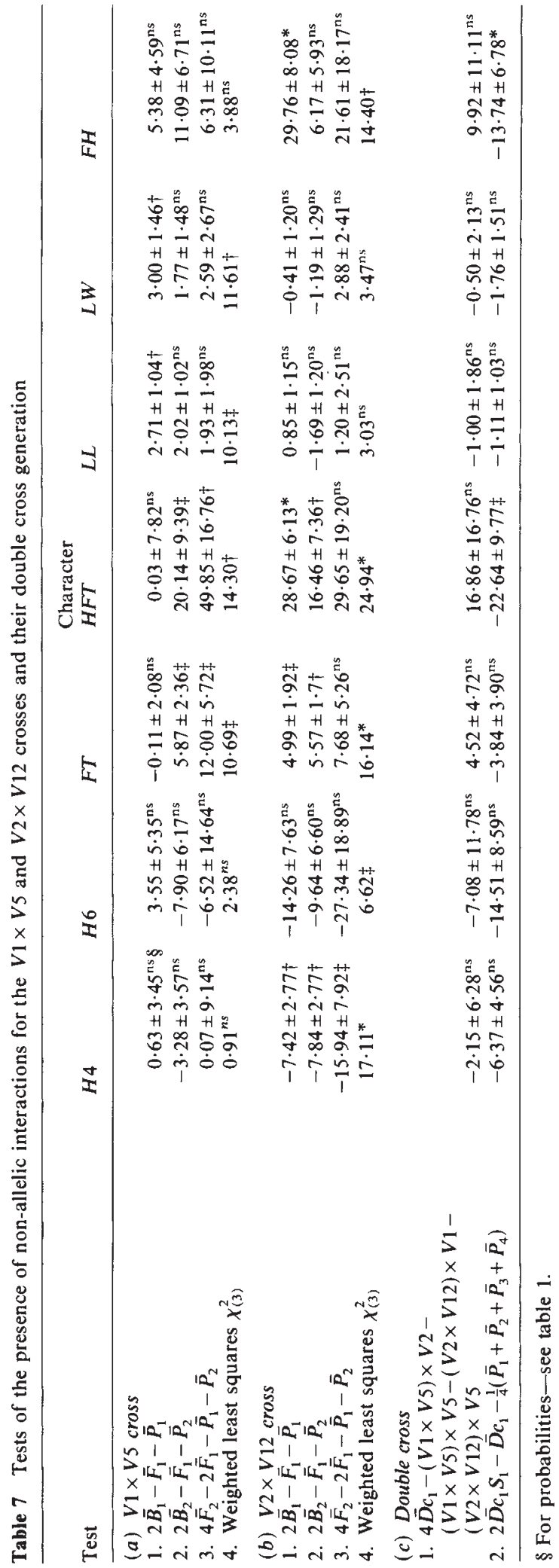


Table 8 Estimates of environmental variance $E_{w}$ of $B, D, B+D$ and $D c$ samples of inbred lines and the tests of homogeneity of these components for each of the seven characters

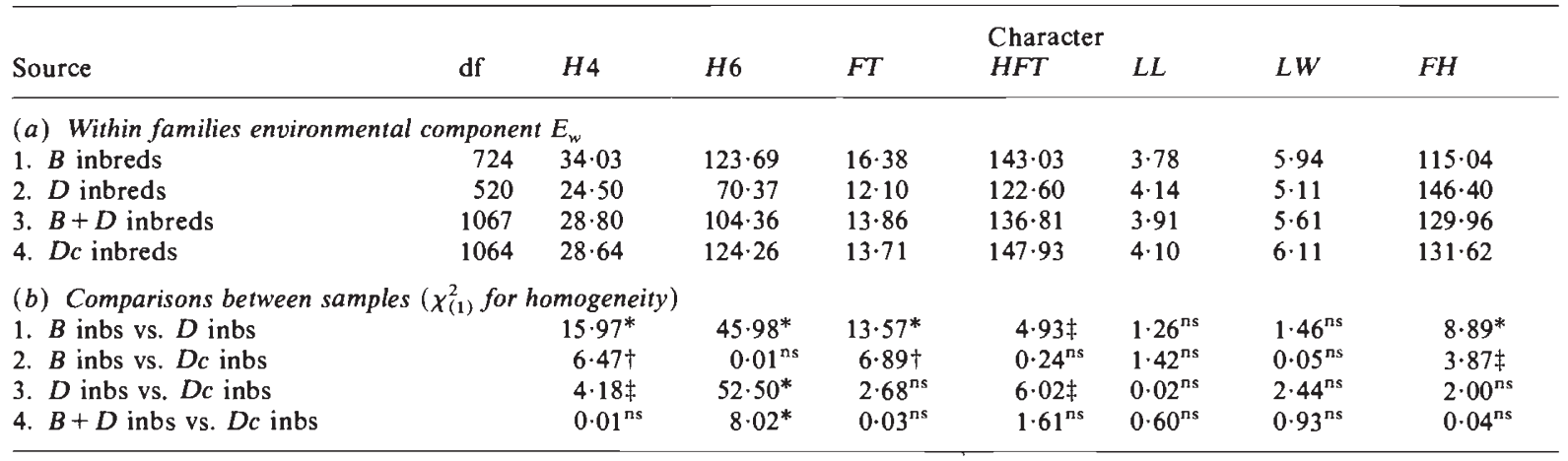

$\S$ For probabilities see table 1.

cross can differ both in the absence and presence of complications like non-allelic interactions, linkage disequilibrium and unequal gene frequencies. However, while the estimates of statistics like mean, variance, skewness, kurtosis and range will differ between $B$ and $D$ samples because they were extracted from diverse genetic stocks, those of $D c$ sample will take different values because $D c$ inbreds are only half related to the $B$ or $D$ inbreds. Further, the magnitudes of these differences are highly influenced by the degree of gene association/dispersion, linkage disequilibrium and the type of epistasis prevailing in the material. Therefore, the differences may be significant only when the effects of these sources are acting in unison.

Reference to section 4 and tables 1 to 10 shows that the experimental results are in complate agreement with the above conclusions. For instance, statistics of the $B$ and $D$ samples often show large differences in magnitude and tests of these deviations are significant on 32 (out of 63) occasions. Statistics of the $D c$ sample, on the other hand, have values that are generally closer to the average of those of the $B$ and $D$ samples. They are even more close in value to the statistics of the combined distributions of $B$ and $D$ samples. Consequently differences between the $D c$ and $B+D$ samples are significant on only four occasions while those between $B$ and $D c$ and $D$ and $D c$ samples are significant on 20 and 25 occasions, respectively.

Further, we can quantify the relative contributions of various groups of loci by establishing the patterns of differences that exist between the samples. For instance, if the same loci are segregating in the single crosses $\left(k b=k^{\mathrm{II}}, k b_{1}=k b_{2}=k f \simeq\right.$ 0 ) and the two pairs of parents differ only for association/dispersion of alleles at these loci then the statistics of the three samples are expected to be equal in magnitude within the limits of their standard errors (see section 2). On the other hand, if none of these loci is common between the single crosses $(k b=0)$ and the parents do not show allelic differences for any of the background loci $(k f=0)$ then differences between samples will be highly significant. More specifically, we expect the additive genetic variance of $D c$ sample to be equal to $\frac{3}{4}$ of the sum of those of $B$ and $D$ samples. Further, depending upon the level of gene association/dispersion and the average frequencies of the alleles over all the loci, the highest and lowest scores

Table 9 Bartlett's tests of homogeneity for the within family variances within different samples of inbred lines for the seven characters

\begin{tabular}{|c|c|c|c|c|c|c|c|c|}
\hline Source & $\mathrm{df}$ & $H_{4}$ & $H 6$ & $F T$ & $\begin{array}{l}\text { Character } \\
H F T\end{array}$ & $L L$ & $L W$ & $F H$ \\
\hline$B$ inbreds & 80 & $187 \cdot 76^{*}$ & $134 \cdot 37^{*}$ & $255 \cdot 25^{*}$ & $224 \cdot 14^{*}$ & $94 \cdot 33^{\mathrm{ns}}$ & $111 \cdot 79 \ddagger$ & $171 \cdot 52^{*}$ \\
\hline$D c$ inbreds & 119 & $307 \cdot 20^{*}$ & $282.73^{*}$ & $399 \cdot 31 *$ & $339 \cdot 13^{*}$ & $229 \cdot 92^{*}$ & $205.97^{*}$ & $252 \cdot 47^{*}$ \\
\hline$B+D$ inbreds & 117 & $327 \cdot 42^{*}$ & $275 \cdot 00^{*}$ & $345 \cdot 28^{*}$ & $337 \cdot 79^{*}$ & $165 \cdot 85^{*}$ & $157 \cdot 47^{*}$ & $312 \cdot 74^{*}$ \\
\hline$D c$ inbreds $\S$ & 117 & $305.93^{*}$ & $270 \cdot 13^{*}$ & $354 \cdot 86^{*}$ & $322 \cdot 42^{*}$ & $225 \cdot 72^{*}$ & $204 \cdot 23^{*}$ & $246 \cdot 79^{*}$ \\
\hline
\end{tabular}

$\S \chi^{2}(117)$ was obtained from within variances of 118 of the $120 \mathrm{Dc}$ inbreds.

For probabilities see table 1 
Table 10 Standard regression analyses for testing the significance of linear dependence of $S D$ 's on mean performance and joint regression analyses for testing the homogeneity of regressions between samples

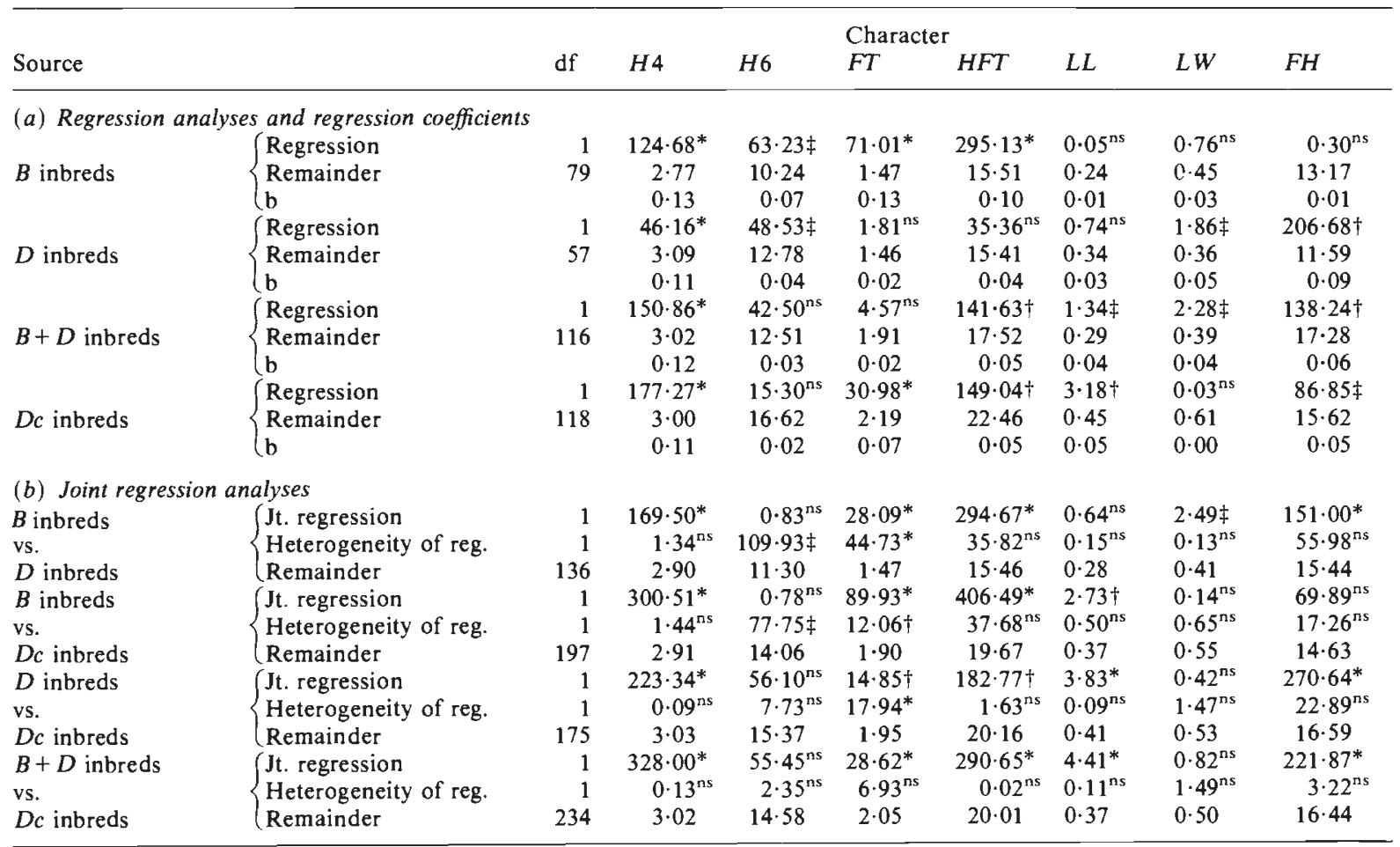

$\S$ For probabilities see table 1.

amongst the $D c$ inbreds should also be the most extreme amongst the three samples. Finally, if the background genes $(k f)$ are the only source of genetic variation $\left(k f \simeq k^{\mathrm{II}} ; k b_{1}=k b_{2}=k b=0\right)$ and the mean differences between the $B$ and $D$ samples are small $\left(r d \sum_{i=1}^{k f} d_{i} \simeq 0\right)$ then the additive genetic variance and the range of $D c$ inbreds should be much larger than those of $B$ and $D$ samples. These relationships, though defined on an additive/dominance model, are also expected to hold when epistasis, linkage disequilibrium and genotype $\times$ micro-environmental interactions are present.

It is apparent from the results that differences between samples are often significant for most of the characters except $L W$ (see tables 1 to 10 ). Also, the statistics of the double cross sample do not take disproportionately larger values for any of the characters. Therefore, while the genetic variability for leaf width may only have resulted from segregation at $k b$ group of loci, most of the other groups $\left(k b_{1}, k b_{2}, k f\right.$, etc.) must also be contributing to the genetic variability that is present for other characters. Further, the preponderance of plus alleles at the $k b_{1}$ and $k b_{2}$ groups of loci together with residual heterozygosity and non-allelic interactions can only be responsible for the asymmetry that exists between the high and low scores of $D c$ inbreds for $H 6, H F T, L L$ and $F H$ (see table 5).

Further, it appears as if none of the samples is unconditionally superior in throwing out extreme recombinant inbreds (see results and table 5). For example, the inbred lines with the smallest score have always originated from the $B$ or $D$ sample and those with the highest score come either from the $D c$ sample or the $D$ sample. However, the double cross sample is latently superior firstly because the extreme inbreds extracted from the double cross always rank the first or the second amongst the three samples and secondly because they cover between $88-109$ per cent of the range which is jointly covered by the $B$ and $D$ samples. It also has the first or the second largest proportion of transgressive segregants that perform better than $\bar{P}_{\text {largest }}$ or poorer than $\bar{P}_{\text {smallest }}$, the high and low scoring varieties among the four parents.

Finally, double crosses are often considered to be superior sources of extreme recombinant 
inbreds solely on the grounds that they provide additional opportunities for recombination between the alleles of four parents. The present study confirms this theoretically but also shows that the chances of recovering these extreme inbreds are remote unless the alleles of like effect are completely dispersed amongst the parental varieties and/or only few loci are segregating in each single cross. Consequently, the choice of a double cross without any evidence for its superior potential can limit the fuller exploitation of the genetic variability that is present in the breeding material.

Inbreeding potentials of various source populations are, however, easily predicted from their early generations using the methods of Jinks and Pooni (1976), Pooni and Jinks (1985) and Pooni, Jinks and Yohannes (1985). These predictions have often proved to be reliable and therefore should be used to discriminate between the potentially superior and inferior crosses.

\section{REFERENCES}

CAVALLI, L. L. 1952. An analysis of linkage in quantitative inheritance. Quantitative Inheritance (eds E. C. R. Rieve and C. H. Waddington), H.M.S.O., London.

EBERHART, S. A. 1964. Theoretical relations among single, three way and double cross hybrids. Biometrics, 20, 522-539.

JINKS, J. L AND PERKINS, J. M. 1972. Predicting the range of inbred lines. Heredity, 28, 399-403.

JINKS, J. L. AND POONI, H. S. 1976. Predicting the properties of recombinant inbred lines derived by single seed descent. Heredity, 36, 253-266.
JINKS, J. L. AND POONI, H. S. 1980. Comparing predictions of mean performance and environmental sensitivity of recombinant inbred lines based upon $F_{3}$ and triple test cross families. Heredity, 45, 305-312.

JINKS, J. L. AND POONI, H. S. 1981. Predicting the properties of pure breeding lines extractable from a cross in the presence of linkage. Heredity, 49, 265-270.

JINKS, J. L. AND POONI, H. S. 1984. Comparison of inbred lines produced by single seed descent and pedigree inbreeding. Heredity, 53, 299-308.

MATHER, K. 1949. Biometrical Genetics (1st Edn.), Methuen, London.

MATHER, K. 1973. Statistical Analysis in Biology. Chapman and Hall, London.

MATHER, K. AND JINKS, J. L. 1982. Biometrical Genetics (3rd Edn.) Chapman and Hall, London.

POONI, H. S. AND JINKS, J. L. 1978. Predicting the properties of recombinant inbred lines derived by single seed descent for two or more characters simultaneously. Heredity, 40, 349-361.

POONI, H. S. AND JINKS, J. L. 1985. Predicting the properties of first cycle inbreds and second cycle hybrids extractable from two, three and four parent crosses. Heredity, 54, 397-411.

POONI, H. S., JINKS, J. L. AND CORNISH, M. A. 1977. The causes and consequences of non-normality in predicting the properties of recombinant inbred lines. Heredity, 38, 329338.

POONI, H. S., JINKS, J. L. AND JAYASEKARA, N. E. M. 1978. An investigation of gene action and genotype $\times$ environment interaction in two crosses of Nicotiana rustica by triple test cross and inbred line analysis. Heredity, 41, 83-92.

POONI, H. S., JINKS, J. L. AND POONI, G. S. 1980. A general method for the detection and estimation of additive, dominance and epistatic variation for metrical traits. IV. Triple test cross analysis for normal families and their selfs. Heredity, 44, 177-192.

POONI, H. S., JINKS, J. L. AND YOHANNES, D. 1985. Predicting the properties of random inbreds and second cycle hybrids using progeny families and hierarchical selfs of a four parent cross. Heredity, 55, 111-119.

SNEDECOR, G. W. AND COCHRAN, W. G. 1974. Statistical Methods. Iowa State University Press, Ames, U.S.A. 